\title{
Quick freezing of unfertilized mouse oocytes using ethylene glycol with sucrose or trehalose
}

\author{
A. A. Rayos, Y. Takahashi, M. Hishinuma and H. Kanagawa \\ Department of Theriogenology, Faculty of Veterinary Medicine, Hokkaido University, \\ Sapporo 060, Japan
}

\begin{abstract}
Unfertilized mouse oocytes were frozen by directly plunging them into liquid nitrogen vapour after equilibration in a freezing medium containing 3 mol ethylene glycol $1^{-1}$ with $0.25 \mathrm{~mol}$ sucrose or trehalose $1^{-1}$ for 5-40 min. After thawing and dilution of the cryoprotectant, oocytes of normal morphology were inseminated in vitro and the effect of equilibration period on the rates of fertilization and development in vitro was examined. Regardless of the equilibration in the freezing medium, no significant difference was observed on the fertilization rate of frozen-thawed oocytes. However, higher fertilization and higher normal fertilization rates were obtained with equilibration in 3 mol ethylene glycol $1^{-1}$ with either 0.25 mol sucrose $1^{-1}$ or trehalose for 20 and 40 min than with 5 and 10 min equilibration. Development rates to two-cell embryos and expanded blastocysts of in vitro fertilized frozen-thawed oocytes that were equilibrated in the freezing medium for 20 and 40 min were significantly higher $(P<0.05$ or $P<0.01)$ than with 5 min equilibration. Development in vivo was assessed by transferring blastocysts derived from unfertilized oocytes frozen by the optimum treatment $(20 \mathrm{~min}$ equilibration in the freezing medium before freezing) into the uterine horns of day 3 pseudopregnant female recipients. The development rate of frozen-thawed oocytes to the blastocyst stage after insemination in vitro was significantly lower than that of the non-frozen control $(P<0.001)$. However, transfer of the blastocysts derived from frozen-thawed oocytes to the uterine horns of the recipients resulted in fetal development and implantation rates similar to those of the control. The overall development rates to fetuses of blastocysts derived from in vitro fertilization of mouse oocytes frozen after 20 min equilibration in 3 mol ethylene glycol l ${ }^{-1}$ with $0.25 \mathrm{~mol}$ sucrose $1^{-1}$ or trehalose were 20.3 and $22.5 \%$, respectively.
\end{abstract}

\section{Introduction}

Success in the cryopreservation of mouse embryos with development to normal young after transfer of embryos to recipients was reported by Whittingham et al. (1972). Since then, several studies have been performed using mouse embryos as experimental models, and the embryos of at least 15 mammalian species have been successfully cryopreserved (Leibo, 1989).

In combination with other reproductive technologies, cryopreservation of oocytes can be used for the management of human infertility, livestock breeding and conservation of rare genetic stocks. Cryopreservation of unfertilized mouse oocytes by slow cooling has been reported by Tsunoda et al. (1976), Parkening et al. (1976), Whittingham (1977), Fuller and Bernard (1984), Glenister et al. (1987) and Ko and Threlfall (1988). However, compared with the success rate obtained with embryos, the development rates of frozen-thawed oocytes are still low.

Received 8 March 1993
Earlier studies on oocyte cryopreservation used the slow or conventional cooling procedures. Attempts at improving the techniques have been directed to the simplification of the freezing procedures. Nakagata (1989), Kono et al. (1991) and Shaw et al. (1991) reported high development rates of vitrified-warmed mouse oocytes after fertilization in vitro. Surrey and Quinn (1990) successfully cryopreserved unfertilized mouse oocytes by a combined process of dehydration of the oocyte with sucrose and permeation with dimethyl sulfoxide before plunging the oocytes into liquid nitrogen (ultrarapid freezing). In the studies of Rayos et al. (1992a, b), early stage mouse embryos were successfully cryopreserved after determining the optimum equilibration period in $3 \mathrm{~mol}$ ethylene glycol $\mathrm{l}^{-1}$ with $0.25 \mathrm{~mol}$ sucrose $1^{-1}$ or lactose before plunging them into liquid nitrogen vapour (quick freezing). Unfertilized mouse oocytes have low permeability to cryoprotectants (Mazur et al., 1976; Jackowski et al., 1980); equilibration in the freezing medium is therefore of critical importance for survival before quick freezing (Szell and Shelton, 1986; Takahashi and Kanagawa, 1990). 
This study was conducted to examine the effect of equilibration period in $3 \mathrm{~mol}$ ethylene glycol $\mathrm{I}^{-1}$ with $0.25 \mathrm{~mol}$ sucrose $1^{-1}$ or trehalose on the post-thaw, post-dilution survival, fertilization in vitro as well as in vivo development rates of unfertilized mouse oocytes cryopreserved by a quick freezing method.

Trehalose, a non-reducing disaccharide of glucose, which has been found to maintain membrane integrity during dehydration (Crowe and Crowe, 1984), was used as a non-permeable cryoprotectant in combination with glycerol in the rapid (Krag et al., 1985) and conventional (Honadel et al., 1988) freezing of early preimplantation mouse embryos. Compared with sucrose, trehalose was also reported by $\mathrm{Kim}$ et al. (1986) to improve the viability of mouse morulae frozen ultrarapidly. The use of trehalose as a cryoprotectant for the cryopreservation of unfertilized oocytes was mentioned briefly by Bernard et al. (1990). This study also compares trehalose with sucrose as a non-permeable cryoprotectant in the quick freezing of unfertilized mouse oocytes.

\section{Materials and Methods}

\section{Experimental animals}

Female $\mathrm{F}_{1}$ hybrid (C57BL/6] $\times \mathrm{CBA}$ ) mice, 6-8 weeks of age and $20-25 \mathrm{~g}$, were used as oocyte donors. Female ICR strain mice, 10-12 weeks of age and 30 to $40 \mathrm{~g}$, were used as recipients. They were maintained in a room with controlled lighting $14 \mathrm{~h}$ light $(05: 00$ to $19: 00 \mathrm{~h}): 10 \mathrm{~h}$ dark cycle and given commercial feed and water ad libitum. Female $\mathrm{F}_{1}$ mice were induced to superovulate by i.p. injections of 5 iu pregnant mares' serum gonadotrophin (PMSG) (Serotrophin: Teikoku Zoki, Tokyo) and 5 iu hCG (Gonatrophin: Teikoku Zoki) given $48 \mathrm{~h}$ apart. Fourteen to fifteen hours after hCG injection, they were killed by cervical dislocation and unfertilized oocytes were released from excised oviducts into modified phosphatebuffered saline (PBI; Whittingham, 1971) supplemented with $10 \%$ fetal bovine serum (FBS; Life Technologies, Inc., Grand Island, $\mathrm{NY})$ and containing hyaluronidase $\left(150 \mathrm{U} \mathrm{ml}^{-1}\right.$; bovine testis type I-S: Sigma Chemical Co., St Louis, MO) for 5-10 min. When the cumulus cells were detached, the oocytes were washed three times in fresh PBI medium with $10 \%$ FBS and pooled in sterile plastic dishes (Nunc, Nunclon, Kamstrup) until used. Only oocytes with normal morphology were used in this study.

\section{Freezing procedure}

A modified freezing method described by Takahashi and Kanagawa (1985, 1990) was used in this study. Briefly, the oocytes were pipetted into a plastic dish containing the freezing medium ( $3 \mathrm{~mol}$ ethylene glycol $\mathrm{l}^{-1}$ with either 0.25 mol sucrose $\mathrm{I}^{-1}$ or trehalose) in PB1 with $10 \%$ FBS. After the oocytes had settled at the bottom of the dish, 10-15 oocytes together with the freezing medium were drawn into a $0.25 \mathrm{ml}$ French straw (IMV, L'Aigle) which was heat sealed and equilibrated for $5,10,20$ and $40 \mathrm{~min}$. The equilibration period included the time the oocytes were in the dish containing the freezing medium. The volume of the freezing medium contain- ing the oocytes was $135 \mu \mathrm{l}$ and was separated by air bubbles on each side and the remainder of the straw was filled with the same medium. After the assigned equilibration period was attained, the oocytes were frozen in liquid nitrogen vapour (about $-170^{\circ} \mathrm{C}$ ), by placing the sealed straw horizontally on a styrofoam plate $(140 \mathrm{~mm} \times 60 \mathrm{~mm} \times 5 \mathrm{~mm})$ with a stainless steel mesh on its upper surface and floating in a liquid nitrogen bath. Two minutes later, the straw was plunged into liquid nitrogen and stored for 1-120 days.

\section{Thawing and dilution}

Thawing was performed in a water bath at $37^{\circ} \mathrm{C}$ for $20 \mathrm{~s}$. The contents of the straw were then expelled into a Petri dish and the oocytes were immediately pipetted into $0.5 \mathrm{~mol} \mathrm{l}^{-1}$ of the same sugar used in the freezing medium for one-step dilution of the cryoprotectant. After $5 \mathrm{~min}$, the oocytes were washed three times with PB1 with 10\% FBS and oocytes with normal morphology were transferred into $0.4 \mathrm{ml}$ of TYH medium (Toyoda et al., 1971) supplemented with $4 \mathrm{mg}$ BSA $\mathrm{ml}^{-1}$ (> $98 \%$ (GE) Sigma Chemical Co.) under paraffin oil for $30 \mathrm{~min}$ in an atmosphere of $5 \% \mathrm{CO}_{2}$ in air at $37^{\circ} \mathrm{C}$ before fertilization in vitro.

\section{Fertilization in vitro}

In vitro fertilization was carried out according to the method of Toyoda et al. (1971). All oocytes with normal morphology after freezing and thawing (surviving oocytes) as well as the non-frozen control, underwent insemination in vitro. Semen was collected from the cauda epididymides, one from each of two mature $\mathrm{F}_{1}(\mathrm{C} 57 \mathrm{BL} / 6 \mathrm{~J} \times \mathrm{CBA})$ males, allowed to disperse in $0.4 \mathrm{ml}$ TYH medium under paraffin oil and incubated in an atmosphere of $5 \% \mathrm{CO}_{2}$ in air at $37^{\circ} \mathrm{C}$ for $30 \mathrm{~min}$. Thereafter, the sperm concentration was determined by means of a haemocytometer and the spermatozoa were incubated for a further $1-1.5 \mathrm{~h}$. A suitable volume $(4-10 \mu \mathrm{l})$ of spermatozoa was added to the medium containing the oocytes and incubated for $6 \mathrm{~h}$, providing a final sperm concentration of 150 cells $\mu \mathrm{l}^{-1}$.

The fertilization rate was assessed by removing frozenthawed as well as the non-frozen control oocytes $6 \mathrm{~h}$ after insemination, mounting them on a slide, fixing with $10 \%$ neutral formalin overnight, washing with $99.5 \%$ ethanol, staining with $0.25 \%$ aceto-lacmoid, and then examining the slides under a phase-contrast microscope for evidence of fertilization. Oocytes were considered as fertilized when they had an enlarged sperm head(s) or male pronucleus(ei) with corresponding sperm tail(s). The frequency of normal fertilization was determined as a ratio of oocytes with a pair of pronuclei and corresponding sperm tail to the total number of oocytes evaluated.

\section{Culture in vitro}

In a separate experiment, frozen-thawed and control oocytes of normal morphology also underwent insemination in vitro as described above. The oocytes were then washed three times with Whitten's medium (Whitten, 1971) supplemented 


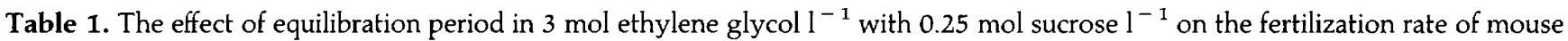
oocytes cryopreserved by a quick freezing method

\begin{tabular}{|c|c|c|c|c|c|}
\hline Equilibration period (min) & $\begin{array}{l}\text { Number of } \\
\text { oocytes frozen }\end{array}$ & $\begin{array}{l}\text { Number (\%) } \\
\text { surviving after } \\
\text { thawing and dilution } \\
\text { and inseminated } \\
\text { in vitro }\end{array}$ & $\begin{array}{l}\text { Number (\%) } \\
\text { fertilized }^{+}\end{array}$ & $\begin{array}{c}\text { Number (\%) } \\
\text { normally } \\
\text { fertilized }^{\ddagger}\end{array}$ & $\begin{array}{c}\text { Overall normal } \\
\text { fertilization rate }(\%)^{\S}\end{array}$ \\
\hline 5 & 142 & $74(52.1)^{a}$ & $57(77.0)^{* *}$ & $42(56.8)^{* * *}$ & $29.6^{a * * *}$ \\
\hline 10 & 125 & $84(67.2)^{\mathrm{b}}$ & $67(79.8)^{* *}$ & $51(60.7)^{* * *}$ & $40.8^{\mathrm{ab} * * *}$ \\
\hline 20 & 133 & $103(77.4)^{\mathrm{b}}$ & $87(84.5)$ & $71(68.9)^{* *}$ & $53.4^{b * * *}$ \\
\hline 40 & 127 & $98(77.2)^{\mathrm{b}}$ & $83(84.7)$ & $66(67.3)^{* *}$ & $52.0^{\mathrm{b} * * *}$ \\
\hline Non-frozen control & & 94 & $88(93.6)$ & $82(87.2)$ & 87.2 \\
\hline
\end{tabular}

\footnotetext{
${ }^{\dagger}$ Number fertilized: number of oocytes inseminated in vitro.

${ }^{\ddagger}$ Number normally fertilized: number of oocytes inseminated in vitro.

${ }_{N}$ Number normally fertilized: number of oocytes frozen.

Values with different superscripts in the same column are significantly different $\left({ }^{\mathrm{ab}} P<0.01\right)$.

Values with asterisks are significantly different from the control $\left.{ }^{* *} P<0.01{ }^{* * *} P<0.001\right)$.
}

with $3 \mathrm{mg} \mathrm{BSA} \mathrm{ml}{ }^{-1} 6 \mathrm{~h}$ after insemination in vitro before they were cultured in $100 \mu \mathrm{l}$ of Whitten's medium with $100 \mu \mathrm{mol}$ EDTA $1^{-1}$ (Kanto Chemical Co., Tokyo) under paraffin oil at $37^{\circ} \mathrm{C}$ in $5 \% \mathrm{CO}_{2}$ in air. After $19 \mathrm{~h}$ of incubation $(24 \mathrm{~h}$ after insemination), the culture was examined under inverted microscope and the number of two-cell embryos was recorded. Development to the expanded blastocyst stage was assessed $110-120 \mathrm{~h}$ after insemination.

All manipulations were performed at room temperature $\left(20-25^{\circ} \mathrm{C}\right)$. Survival of the frozen-thawed oocytes was assessed at the following level: proportion of oocytes of normal morphology after thawing and dilution, development to two-cell embryos and to expanded blastocysts.

\section{Parthenogenetic activation}

Parthenogenetic activation of oocytes after exposure to a cryoprotectant, cooling, thawing and dilution has been reported by Tarkowski (1975), Shaw and Trounson (1989) and Kono et al. (1991). Oocytes frozen after equilibration in $3 \mathrm{~mol}$ ethylene glycol $1^{-1}$ with $0.25 \mathrm{~mol}$ sucrose $1^{-1}$ or trehalose for 5-40 min were incubated in TYH medium for $6 \mathrm{~h}$ without sperm and cultured in Whitten's medium for 4 days, to determine whether oocyte activation occurs after quick freezing and thawing.

\section{Development in vivo}

Blastocysts derived from oocytes frozen by the optimum treatment ( $20 \mathrm{~min}$ equilibration in $3 \mathrm{~mol}$ ethylene glycol $1^{-1}$ with either 0.25 mol sucrose $1^{-1}$ or trehalose) were transferred into the uterine horns of day 3 pseudopregnant female ICR recipients (six to nine embryos per horn) to assess viability in vivo. The recipients were killed on day 18 of pregnancy, and the number of live and resorbing fetuses, as well as the implantation sites were determined. Blastocysts developing from non-frozen oocytes after insemination and culture in vitro were also transferred into the uterine horns of the recipients to serve as controls.

\section{Statistical analysis}

Experiments were repeated four times and data on survival after thawing and dilution, fertilization in vitro, and development in vitro and in vivo of the frozen-thawed oocytes were analysed by $\chi^{2}$ test.

\section{Results}

\section{Morphology of oocytes after thawing and dilution}

More than $90 \%$ of the frozen oocytes were recovered after thawing and no significant difference was observed in the post-thaw recovery, regardless of the equilibration period. After thawing and dilution, the number of oocytes of normal morphology frozen after 10,20 or $40 \mathrm{~min}$ equilibration in $3 \mathrm{~mol}$ ethylene glycol $1^{-1}$ with either $0.25 \mathrm{~mol}$ sucrose $1^{-1}$ or trehalose was significantly higher $(P<0.01)$ than with 5 min equilibration (Tables 1-4). No significant difference on the survival (normal morphology) of frozen mouse oocytes after thawing and dilution was observed irrespective of the sugar (sucrose or trehalose) used in combination with ethylene glycol.

\section{Fertilization rate}

No significant difference on the fertilization rate among mouse oocytes equilibrated in $3 \mathrm{~mol}$ ethylene glycol I $\mathrm{I}^{-1}$ with either $0.25 \mathrm{~mol}$ sucrose $1^{-1}$ or $0.25 \mathrm{~mol}$ trehalose $\mathrm{l}^{-1}$ before plunging into liquid nitrogen vapour was observed (Tables 1 and 2). However, higher fertilization and higher normal fertilization rates were obtained with 20 and 40 min equilibration in the freezing medium than with 5 or $10 \mathrm{~min}$ equilibration. Moreover, the fertilization rates of the oocytes cryopreserved after 20 and 40 min equilibration were not significantly different from the control value, while the fertilization rates of oocytes cryopreserved after 5 or $10 \mathrm{~min}$ in the freezing medium were significantly lower $(P<0.01)$ than those of the controls. Most of the frozen-thawed oocytes that had 
Table 2. The effect of equilibration period in 3 mol ethylene glycol $1^{-1}$ with 0.25 mol trehalose $1^{-1}$ on the fertilization rate of mouse oocytes cryopreserved by a quick freezing method

\begin{tabular}{|c|c|c|c|c|c|}
\hline Equilibration period (min) & $\begin{array}{l}\text { Number of } \\
\text { oocytes frozen }\end{array}$ & $\begin{array}{c}\text { Number }(\%) \\
\text { surviving after } \\
\text { thawing and dilution } \\
\text { and inseminated } \\
\text { in vitro }\end{array}$ & $\begin{array}{c}\text { Number }(\%) \\
\text { fertilized }^{\dagger}\end{array}$ & $\begin{array}{c}\text { Number }(\%) \\
\text { normally fertilized }\end{array}$ & $\begin{array}{c}\text { Overall normal } \\
\text { fertilization rate }(\%)^{\S}\end{array}$ \\
\hline 5 & 123 & $68(55.3)^{a}$ & $53(77.9)^{* *}$ & $35(55.9)^{* * *}$ & $30.9^{a * * *}$ \\
\hline 10 & 114 & $76(66.7)^{\mathrm{ab}}$ & $59(77.6)^{* *}$ & $45(59.2)^{* * *}$ & $39.5^{\mathrm{ab} * * *}$ \\
\hline 20 & 101 & $72(71.3)^{b}$ & $62(86.1)$ & $50(69.4)^{* *}$ & $49.5^{\mathrm{b} * * *}$ \\
\hline 40 & 124 & $38(71.0)^{\mathrm{b}}$ & $75(85.1)$ & $60(68.4)^{* *}$ & $48.4^{\mathrm{b} * * *}$ \\
\hline Non-frozen control & & 94 & $88(93.6)$ & $82(87.2)$ & 87.2 \\
\hline
\end{tabular}

${ }^{\dagger}$ Number fertilized: number of oocytes inseminated in vitro.

${ }^{\ddagger}$ Number normally fertilized: number of oocytes inseminated in vitro.

${ }^{\$}$ Number normally fertilized: number of oocytes frozen.

Values with different superscripts in the same column are significantly different $\left({ }^{\mathrm{ab}} P<0.01\right)$.

Values with asterisks are significantly different from the control $\left({ }^{* *} P<0.01 ; * * P<0.001\right)$.

Table 3. The effect of equilibration period in $3 \mathrm{~mol}$ ethylene glycol $1^{-1}$ with $0.25 \mathrm{~mol}$ sucrose $1^{-1}$ on the in vitro development of mouse oocytes cryopreserved by a quick freezing method

\begin{tabular}{|c|c|c|c|c|c|}
\hline Equilibration period (min) & $\begin{array}{c}\text { Number of } \\
\text { oocytes frozen }\end{array}$ & $\begin{array}{l}\text { Number }(\%) \\
\text { surviving after } \\
\text { thawing and dilution } \\
\text { and inseminated } \\
\text { in vifro }\end{array}$ & $\begin{array}{l}\text { Number }(\%) \\
\text { developed to } \\
\text { two-cell }^{\dagger}\end{array}$ & $\begin{array}{l}\text { Number }(\%) \\
\text { developed to } \\
\text { expanded } \\
\text { blastocyst }^{\ddagger}\end{array}$ & $\begin{array}{l}\text { Overall rate } \\
\text { of development } \\
\text { to expanded } \\
\text { blastocyst }(\%)^{\S}\end{array}$ \\
\hline 5 & 216 & $110(50.9)^{a}$ & $65(59.1)^{c}$ & $52(47.3)^{\mathrm{a}}$ & $24.1^{\mathrm{a}}$ \\
\hline 10 & 204 & $148(72.5)^{\mathrm{b}}$ & $95(64.2)^{\mathrm{cd}}$ & $77(52.0)^{\mathrm{ab}}$ & $37.7^{\mathrm{b}}$ \\
\hline 20 & 220 & $169(76.8)^{b}$ & $118(69.8)^{d}$ & $99(58.6)^{\mathrm{ab}}$ & $45.0^{\mathrm{b}}$ \\
\hline 40 & 225 & $172(76.4)^{\mathrm{b}}$ & $122(70.9)^{d}$ & $100(58.1)^{\mathrm{b}}$ & $44.4^{\mathrm{b}}$ \\
\hline Non-frozen control & & 161 & $146(90.7)^{* * *}$ & $141(87.6)^{* * *}$ & $87.6^{* * *}$ \\
\hline
\end{tabular}

\footnotetext{
${ }^{\dagger}$ Number developed to two-cell embryos: number of oocytes inseminated in vitro.

${ }_{\text {}}$ Number developed to expanded blastocysts: number of oocytes inseminated in vitro.

\$ Number developed to expanded blastocysts: number of oocytes frozen.

Values with different superscripts in the same column are significantly different $\left({ }^{\mathrm{ab}} P<0.01 ;{ }^{\mathrm{cd}} P<0.05\right)$.

Values in the same column without asterisks are significantly different from the control $(* * * P<0.001)$.
}

undergone fertilization were normally fertilized, possessing a pair of pronuclei, but their normal fertilization rate was significantly lower than that of the controls $(P<0.01$ or $P<0.001$ ). Moreover, the rate of polyploidy of the frozenthawed oocytes was significantly higher than in the controls $(P<0.01)$. The highest overall fertilization rate was obtained with 20 min equilibration in $3 \mathrm{~mol}$ ethylene glycol $\mathrm{l}^{-1}$ with either $0.25 \mathrm{~mol}$ sucrose $1^{-1}(53.4 \%)$ or $0.25 \mathrm{~mol}$ trehalose $1^{-1}$ $(49.5 \%)$.

\section{Development in vitro}

Development rates to two-cell stage embryos of in vitro inseminated frozen-thawed oocytes that were equilibrated in the freezing medium for 20 or 40 min were significantly higher $(P<0.05)$ than with 5 min equilibration (Tables 3 and 4 ).
However, development of oocytes equilibrated in the freezing medium for $10 \mathrm{~min}$ before quick freezing to two-cell embryos was not significantly different from development after 5, 20 or $40 \mathrm{~min}$ equilibration. Similarly, development to the expanded blastocyst stage of oocytes equilibrated for 20 or $40 \mathrm{~min}$ in 3 mol ethylene glycol $1^{-1}$ with either 0.25 mol sucrose $1^{-1}$ $(P<0.01)$ or $0.25 \mathrm{~mol}$ trehalose $\mathrm{l}^{-1}(P<0.05)$ before quick freezing was significantly higher than with equilibration for $5 \mathrm{~min}$. However, a high percentage $(82-85 \%)$ of two-cell embryos obtained after insemination in vitro of frozen-thawed oocytes subsequently developed to the expanded blastocyst stage, regardless of the equilibration period. Development of the control oocytes after insemination in vitro to two-cell embryos and expanded blastocysts was significantly higher $(P<0.001)$ than for the frozen-thawed oocytes. The highest overall development rates to expanded blastocysts were attained when oocytes were frozen after equilibration for 
Table 4. The effect of equilibration period in $3 \mathrm{~mol}$ ethylene glycol $1^{-1}$ with $0.25 \mathrm{~mol}$ trehalose $1^{-1}$ on the in vitro development of mouse oocytes cryopreserved by a quick freezing method

\begin{tabular}{|c|c|c|c|c|c|}
\hline Equilibration period (min) & $\begin{array}{l}\text { Number of } \\
\text { oocytes frozen }\end{array}$ & $\begin{array}{l}\text { Number }(\%) \\
\text { surviving after } \\
\text { thawing and dilution } \\
\text { and inseminated } \\
\text { in vitro }\end{array}$ & $\begin{array}{l}\text { Number (\%) } \\
\text { developed to } \\
\text { two-cell }^{\dagger}\end{array}$ & $\begin{array}{c}\text { Number (\%) } \\
\text { developed to } \\
\text { expanded } \\
\text { blastocyst }^{\ddagger}\end{array}$ & $\begin{array}{l}\text { Overall rate } \\
\text { of development } \\
\text { to expanded } \\
\text { blastocyst }(\%)^{\S}\end{array}$ \\
\hline 5 & 225 & $119(52.9)^{\mathrm{a}}$ & $70(58.8)^{c}$ & $61(51.3)^{\mathrm{c}}$ & $27.1^{\mathrm{a}}$ \\
\hline 10 & 224 & $158(70.5)^{b}$ & $106(67.1)^{\mathrm{cd}}$ & $83(52.5)^{\mathrm{cd}}$ & $37.1^{\mathrm{ab}}$ \\
\hline 20 & 227 & $153(67.4)^{\mathrm{b}}$ & $112(73.2)^{\mathrm{d}}$ & $98(64.1)^{e}$ & $43.2^{\mathrm{b}}$ \\
\hline 40 & 230 & $152(66.1)^{\mathrm{b}}$ & $117(77.0)^{\mathrm{d}}$ & $96(63.2)^{\text {de }}$ & $41.7^{\mathrm{b}}$ \\
\hline Non-frozen control & & 161 & $146(90.7)^{* * *}$ & $141(87.6)^{* * *}$ & $87.6^{* * *}$ \\
\hline
\end{tabular}

${ }^{\dagger}$ Number developed to two-cell embryos: number of oocytes inseminated in vitro.

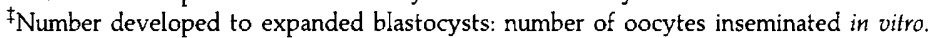

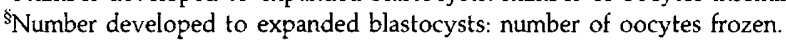

Values with different superscripts in the same column are significantly different $\left({ }^{\mathrm{ab}} P<0.01\right.$; $\left.{ }^{\mathrm{cde}} P<0.05\right)$.

Values in the same column without asterisks are significantly different from the control $\left({ }^{* * *} P<0.001\right)$.

Table 5. In vivo development of mouse oocytes quickly frozen using $3 \mathrm{~mol}$ ethylene glycol $\mathrm{l}^{-1}$ with $0.25 \mathrm{~mol}$ sucrose $\mathrm{l}^{-1}$ or trehalose

\begin{tabular}{|c|c|c|c|c|c|}
\hline Group & $\begin{array}{l}\text { Number of } \\
\text { oocytes frozen }\end{array}$ & $\begin{array}{c}\text { Number (\%) } \\
\text { surviving after } \\
\text { thawing and dilution } \\
\text { and inseminated } \\
\text { in vifro }\end{array}$ & $\begin{array}{l}\text { Number (\%) } \\
\text { developed to } \\
\text { blastocysts and } \\
\text { transferred to } \\
\text { recipients }^{\dagger}\end{array}$ & $\begin{array}{l}\text { Number }(\%) \\
\text { of fetuses }\end{array}$ & $\begin{array}{c}\text { Overall fetal } \\
\text { development } \\
\text { rate }(\%)^{\S}\end{array}$ \\
\hline \multicolumn{6}{|l|}{ Frozen-thawed } \\
\hline Sucrose & 202 & $134(66.3)$ & $78(58.2)^{\mathrm{a}}$ & $41(52.6)$ & $20.3^{\mathrm{a}}$ \\
\hline Trehalose & 215 & $148(68.8)$ & $84(56.8)^{\mathrm{a}}$ & $44(52.4)$ & $20.5^{\mathrm{a}}$ \\
\hline Non-frozen control & & 130 & $112(86.2)^{\mathrm{b}}$ & $63(56.3)$ & $48.5^{\mathrm{b}}$ \\
\hline
\end{tabular}

${ }^{\dagger}$ Number of blastocysts: number of surviving oocytes inseminated in vitro.

${ }^{\ddagger}$ Number of live fetuses: number of blastocysts transferred.

$\$$ Number of live fetuses: number of oocytes frozen.

${ }^{\mathrm{ab}} \mathrm{P}<0.001$.

$20 \mathrm{~min}$ in $3 \mathrm{~mol}$ ethylene glycol $\mathrm{I}^{-1}$ with either $0.25 \mathrm{~mol}$ sucrose $1^{-1}(45.0 \%)$ or $0.25 \mathrm{~mol}$ trehalose $1^{-1}(43.2 \%)$.

\section{Parthenogenetic activation}

Parthenogenetic activation or cleavage of frozen-thawed oocytes, irrespective of the freezing medium used $(3 \mathrm{~mol}$ ethylene glycol $1^{-1}$ with either $0.25 \mathrm{~mol}$ sucrose $1^{-1}$ or $0.25 \mathrm{~mol}$ trehalose $\mathrm{I}^{-1}$ ) and the equilibration period, was not observed following 4 days of culture in Whitten's medium.

\section{Development in vivo}

The development in vivo of unfertilized mouse oocytes quickly frozen after $20 \mathrm{~min}$ equilibration in $3 \mathrm{~mol}$ ethylene glycol $1^{-1}$ with either $0.25 \mathrm{~mol}$ sucrose $\mathrm{I}^{-1}$ or $0.25 \mathrm{~mol}$ trehalose $1^{-1}$ is shown (Table 5). Development of frozenthawed oocytes after insemination and culture in vitro to the blastocyst stage was significantly lower than in the controls
$(P<0.001)$. Blastocysts derived from frozen-thawed and nonfrozen control oocytes were transferred into the uterine horns of pseudopregnant recipients. The proportions of the blastocysts derived from frozen-thawed oocytes that developed to 18-day live fetuses (52.6 and $52.4 \%$ ) were similar to the control values $(56.8 \%)$. Moreover, the implantation rates between the blastocysts derived from frozen-thawed oocytes were not significantly different from the control $(79.5$ and $82.1 \%$ versus $85.7 \%$ ). The overall rates of development to fetus of blastocysts derived from in vitro fertilization of mouse oocytes frozen after $20 \mathrm{~min}$ equilibration in $3 \mathrm{~mol}$ ethylene glycol $\mathrm{I}^{-1}$ with $0.25 \mathrm{~mol}$ sucrose $\mathrm{l}^{-1}$ or $0.25 \mathrm{~mol}$ trehalose $1^{-1}$ were 20.3 and $20.5 \%$, respectively.

\section{Discussion}

For the first time, unfertilized mouse oocytes were successfully frozen by a combined process of dehydration of the oocytes with sucrose or trehalose and permeation with ethylene glycol before plunging them into liquid nitrogen vapour. 
The study reported here also confirmed reports of Szell and Shelton (1986), Takahashi and Kanagawa (1990) and Mazur (1990) that equilibration in a freezing medium containing high concentrations of cryoprotectant is of critical importance for the survival of embryos or oocytes (Surrey and Quinn, 1990) frozen at an extremely rapid rate than it is for those frozen by slow freezing. Abas et al. (1990) and Rayos et al. (1992a, b) indicated that optimum survival can be attained with $5 \mathrm{~min}$ equilibration in $3 \mathrm{~mol}$ ethylene glycol $1^{-1}$ with $0.25 \mathrm{~mol}$ sucrose $\mathrm{I}^{-1}$ or $0.25 \mathrm{~mol}$ lactose $\mathrm{I}^{-1}$ for mouse morula, and $10 \mathrm{~min}$ for one-, two-, four- and eight-cell embryos, thus allowing sufficient time for the cryoprotectant to permeate into the embryonic cells. The study reported here showed that equilibration of unfertilized mouse oocytes for 20 or $40 \mathrm{~min}$ in the freezing medium before freezing results in a high proportion of oocytes of normal morphology, fertilization and development to two-cell embryos and blastocysts after thawing and dilution. The need for longer equilibration in the freezing medium as a requisite for high survival of mouse oocytes could be explained by the different permeabilities of fertilized and unfertilized oocytes (Jackowski et al., 1980). The permeability of unfertilized oocytes to the cryoprotectant is low, but increases as development proceeds to the blastocyst stage after fertilization (Mazur et al., 1976; Schneider and Mazur, 1984). The time required for embryos $(5-10 \mathrm{~min}$ ) may be insufficient for the permeation of the cryoprotectant into the oocytes to the extent that the cryoprotective agent can exert its protective effect. Although most of the frozen oocytes were of normal morphology after thawing, more oocytes frozen after short ( 5 min) equilibration may have suffered membrane damage that was not evident under the dissecting microscope. Thus, the number of degenerated frozen-thawed oocytes increased after dilution of the cryoprotectant.

Smorag et al. (1990) reported that trehalose has a better stabilizing effect on the cell membranes of one- and two-cell rabbit embryos than does sucrose and results in improved viability. Moreover, mouse morula equilibrated in glyceroltrehalose solution before plunging into liquid nitrogen had a higher development rate to expanded blastocysts than did those equilibrated in glycerol-sucrose solution ( $\mathrm{Kim}$ et al., 1986). In our study, however, no significant difference was observed on the survival, fertilizability and developmental capacity of frozen-thawed oocytes, regardless of the sugar used. This finding indicates that sucrose or trehalose in combination with ethylene glycol are effective in the quick freezing of unfertilized oocytes. Observations by Heyman et al. (1986) did not demonstrate the beneficial effect of trehalose in the rapid freezing of bovine non-mature oocytes and one-cell embryos and viability remained at the level obtained with sucrose. Thus, the possible stabilizing effect of trehalose on the cell membranes may be species- and cell-stage dependent.

Unfertilized mouse oocytes cryopreserved after $20 \mathrm{~min}$ equilibration in the freezing medium had a higher rate of normal fertilization than did 5,10 or $40 \mathrm{~min}$ of equilibration. This finding was similar to the proportion of surviving frozen-thawed oocytes that developed to two-cell embryos $24 \mathrm{~h}$ after insemination and culture in vitro. However, subsequent development in vitro to expanded blastocysts of two-cell embryos derived from frozen-thawed oocytes was similar, regardless of the equilibration period. This finding shows that a high proportion of frozen-thawed oocytes that undergo normal fertilization can develop into two-cell embryos and subsequently into expanded blastocysts.

The fertilization and development rates of frozen-thawed oocytes were significantly lower than those of the controls, indicating that some membrane damage may have occurred during freezing because of intracellular ice formation and thawing, which could have interferred in the subsequent fertilization and development of the oocyte (Whittingham, 1977). Membrane damage may result from osmotic forces, including overexpansion of the cell membranes after loss of surface area during dehydration (Steponkus and Weist, 1979) and disruption of membrane integrity by mass movement of water (Muldrew and McGann, 1990). Failure of fertilization may also be due to a still undefined modification to the zona pellucida or the vitelline membrane during freezing and thawing which inhibits the entry of spermatozoa (Carroll et al., 1989, 1990; Wood et al., 1992).

An increased frequency of polyploidy has been reported by Carroll et al. (1989) in frozen-thawed oocytes compared with the non-frozen controls. Their findings showed that this was caused mainly by the retention of the second polar body and not by polyspermy. In contrast, Kono et al. (1991) reported that the increased incidence of polyploidy was due mainly to polyspermic fertilization in vitrified-warmed oocytes compared with the controls. This was in agreement with our present study in which the incidence of polyspermy, as indicated by the presence of two or more male pronuclei or enlarged sperm head(s), was significantly higher than in the controls.

During normal fertilization, fusion of a spermatozoon with the vitelline membrane of the oocyte leads to a cortical granule reaction. Sperm-specific receptors on the zona pellucida are inactivated by the constituents of the cortical granules and the molecular structure of the zona pellucida is altered. The vitelline membrane also becomes structurally changed by the reaction and these modifications block further sperm penetration. Metabolic changes brought about by freezing or thawing might cause the failure of the normal cortical granule reaction and allow polyspermic fertilization (Glenister et al., 1987; Wood et al., 1992). Moreover, physical damage to the vitelline membrane or to the zona pellucida during freezing and thawing may enable more than one spermatozoa to enter the egg (Whittingham and Adams, 1976).

There was no indication that any procedure involved in the freezing and thawing in the study reported here stimulated parthenogenetic activation. This finding was in agreement with reports by Whittingham (1977) and Shaw et al. (1991), who used conventional and vitrification methods, respectively. However, this result was in contrast to studies of Kono et al. (1991), who reported 2-22\% parthenogenetic activation in vitrified-warmed oocytes.

Live 18-day normal fetuses were obtained after transfer of blastocysts derived from frozen-thawed oocytes that were inseminated and cultured in vitro at a rate similar to the control. Moreover, the implantation rates between the blastocysts obtained from frozen-thawed oocytes and the controls were not significantly different. Acceptable rates of oocyte survival, fertilizability in vitro and embryonic development in vitro and in vivo were obtained in this study and are comparable or superior to those of oocytes frozen by conventional (Tsunoda et al., 
1976; Parkening et al., 1976; Whittingham, 1977; Fuller and Bernard, 1984; Glenister et al., 1987; Ko and Threlfall, 1988) or ultrarapid (Surrey and Quinn, 1990) freezing methods. However, our rates were lower than those from studies in which oocytes were cryopreserved by vitrification (Nakagata, 1989; Kono et al., 1991; Shaw et al., 1991).

The study reported here demonstrated that ethylene glycol in combination with either sucrose or trehalose can be used effectively in the quick freezing of unfertilized mouse oocytes. The quick freezing technique represents an efficient, inexpensive and viable option for the cryopreservation of mouse oocytes.

This study was supported in part by a Grant-in-Aid for Scientific Research (No. 02556039) from the Ministry of Education, Science and Culture, Japan.

\section{References}

Abas Mazni O, Valdez CA, Takahashi Y, Hishinuma M and Kanagawa H (1990) Quick freezing of mouse embryos using ethylene glycol with lactose or sucrose Animal Reproduction Science 22 161-169

Bernard AG, Fuller BJ and Shaw RW (1990) The effect of trehalose on recovery rates after cryopreservation and fertilisation of murine and human oocytes Cryobiology 27 631, Abstract

Carroll J, Warnes GM and Matthews CD (1989) Increase in digyny explains polyploidy after in vitro fertilization of frozen-thawed mouse oocytes Journal of Reproduction and Fertility 85 489-494

Carroll J, Depypere H and Matthews CD (1990) Freeze-thaw induced changes in the zona pellucida explains decreased rates of fertilization in frozen-thawed mouse oocytes Journal of Reproduction and Fertility 90 547-553

Crowe JH and Crowe LM (1984) Preservation of membranes in anhydrobiotic organisms: the role of trehalose Science 223 701-703

Fuller BJ and Bernard AG (1984) Successful in vitro fertilisation of mouse oocytes after cryopreservation using glycerol Cryo-Letters 5 307-312

Heyman Y, Smorag Z, Katska L and Vincent C (1986) Influence of carbohydrate, cooling and rapid freezing on the viability of bovine non-mature oocytes or I-cell fertilised eggs Cryo-Letters 7 170-183

Honadel TE and Killian GJ (1988) Cryopreservation of murine embryos with trehalose and glycerol Cryobiology 25 331-337

Glenister DH, Wood MJ and Kirby C (1987) Incidence of chromosome anomalies in first-cleavage mouse embryos obtained from frozen-thawed oocytes fertilised in vitro Gamete Research 16 205-216

Jackowski S, Leibo SP and Mazur P (1980) Glycerol permeabilities of fertilised and unfertilised mouse ova Journal of Experimental Zoology 211 329-341

Kim SH, Chung KM, Lee CK and Im KS (1986) Effect of trehalose as a non-permeable cryoprotectant on the survival of mouse morula frozenthawed ultrarapidly Korean Journal of Animal Science 31 768-773

Ko $Y$ and Threlfall WR (1988) The effects of 1,2-propanediol as a cryoprotectant in the freezing of mouse oocytes Theriogenology 29 987-995

Kono T, Kwon OY and Nakahara T (1991) Development of vitrified mouse oocytes after in vitro fertilisation Cryobiology 28 50-54

Krag KT, Koeler IM and Wright RW (1985) Trehalose: a non-permeable cryoprotectant for direct freezing of early mouse embryos Theriogenology 23 200 (Abstract)

Leibo SP (1989) Equilibration and non-equilibration cryopreservation of embryos Theriogenology 31 85-93

Mazur P (1990) Equilibrium, quasi-equilibrium, and nonequilibrium freezing of mammalian embryos Cell Biophysics 17 53-92

Mazur P, Rigopoulos N, Jackowski SC and Leibo SP (1976) Preliminary estimates of permeability of mouse and early embryos to glycerol Biophysics journal 16 232 (Abstract)
Muldrew K and McGann LE (1990) Mechanism of intracellular ice formation Biophysics Journal 57 525-532

Nakagata N (1989) High survival rate of unfertilized mouse oocytes after vitrification Joumal of Reproduction and Fertility 87 479-483

Parkening TA, Tsunoda $Y$ and Chang M (1976) Effects of various low temperature, cryoprotective agents and cooling rates on survival, fertilisability and development of frozen-thawed mouse eggs Joumal of Experimental Zoology 197 369-374

Rayos AA, Takahashi Y, Hishinuma M and Kanagawa H (1992a) Quick freezing of one-cell mouse embryos using ethylene glycol with sucrose Theriogenology 37 595-603

Rayos AA, Takahashi Y, Hishinuma M and Kanagawa H (1992b) Quick freezing of mouse two-, four- and eight-cell embryos with ethylene glycol plus sucrose or lactose: effects of developmental stage and equilibration on the survival in vitro Animal Reproduction Science 27 239-245

Schneider U and Mazur P (1984) Osmotic consequences of cryoprotectant permeability and its relation to the survival of frozen-thawed embryos Theriogenology 21 69-79

Shaw JM and Trounson AO (1989) Parthenogenetic activation of unfertilised mouse oocytes by exposure to 1,2-propanediol is influenced by temperature, oocyte age and cumulus removal Gamete Research 24 269-277

Shaw PW, Fuller BJ, Bernard A and Shaw RW (1991) Vitrification of mouse oocytes: improvement rates of survival, fertilisation and development to blastocysts Molecular Reproduction and Development 29 373-378

Smorag Z, Heyman Y, Garnier V and Gajda B (1990) The effect of sucrose and trehalose on the viability of one- and two-cell rabbit embryos Theriogenology 33 741-747

Steponkus PL and Wiest SC (1979) Freeze-thaw induced lesions in the plasma membrane Low Temperature Stress in Crop Plants pp 221-254 Eds JM Lyons, D Graham and JK Raison. Academic Press, London

Surrey ES and Quinn PJ (1990) Successful ultrarapid freezing of unfertilised oocytes Joumal of In Vitro Fertilisation and Embryo Transfer 7 262-266

Szell A and Shelton JN (1986) Role of equilibration before rapid freezing of mouse embryos Journal of Reproduction and Fertility $\mathbf{7 8} 699-703$

Takahashi $Y$ and Kanagawa $H$ (1985) Quick freezing of mouse embryos by direct plunging into liquid nitrogen vapour: effect of sugars Japanese Journal of Veterinary Research 33 141-144

Takahashi $Y$ and Kanagawa $H$ (1990) Effect of equilibration period on the viability of frozen-thawed mouse embryos after rapid freezing Molecular Reproduction and Development 26 105-110

Toyoda Y, Yokoyama M and Hoshi T (1971) Studies on the fertility of mouse eggs in vitro: in vitro fertilisation of mouse eggs by epididymal sperm Japanese Journal of Animal Reproduction 6 147-151

Tsunoda Y, Parkening TA and Chang MC (1976) In vitro fertilisation of mouse and hamster eggs after freezing and thawing Experientia 32 223-224

Tarkowski AK (1975) Induced parthenogenesis in the mouse. In The Development Biology of Reproduction Vol. II pp 93-129 Eds CL Markert and J Papaconstantinous. Academic Press, New York

Whitten WK (1971) Nutrient requirement for cultivation of preimplantation embryos in vitro Advances in Biosciences $6129-141$

Whittingham DG (1971) Survival of mouse embryos after freezing and thawing Nature 223 125-126

Whittingham DG (1977) Fertility in vitro and development to term of unfertilized mouse oocytes previously stored at $-196^{\circ} \mathrm{C}$ Journal of Reproduction and Fertility 49 395-402

Whittingham DG and Adams CE (1976) Low temperature preservation of rabbit embryos Journal of Reproduction and Fertility 47 269-274

Whittingham DG, Leibo SP and Mazur P (1972) Survival of mouse embryos frozen to $-196^{\circ} \mathrm{C}$ and $-296^{\circ} \mathrm{C}$ Science $187411-414$

Wood MJ, Whittingham DG and Lee SH (1992) Fertility failure is not due to premature cortical granule release Biology of Reproduction 46 1187-1195 